\title{
GROWTH OF POLYNOMIALS WITH ZEROS OUTSIDE A CIRCLE
}

\author{
ABDUL AZIZ AND Q. G. MOHAMMAD
}

ABSTRACT. Let $P(z)$ be a polynomial of degree $n$ having all its zeros in $|z|>k>1$. For $k=1$, it is known that

$$
\underset{|z|=R>1}{\operatorname{Max}}|P(z)|<\frac{R^{n}+1}{2} \underset{|z|=1}{\operatorname{Max}}|P(z)| .
$$

In this paper we consider the case $k>1$ and obtain a sharp result.

Let $P(z)$ be a polynomial of degree $n$ such that $\operatorname{Max}_{|z|-1}|P(z)|=1$, then

$$
\underset{|z|=R>1}{\operatorname{Max}}|P(z)|<R^{n} .
$$

Inequality (1) is a simple deduction from the Maximum Modulus Principle (see [4, p. 346] or [3, Vol. I, p. 137, problem III 269]). It was conjectured by Erdös and first proved by Lax [2] that, if $P(z) \neq 0$ in $|z|<1$ then

$$
\underset{|z|=1}{\operatorname{Max}}\left|P^{\prime}(z)\right|<n / 2 \text {. }
$$

Ankeny and Rivlin [1] used (2) to prove the following theorem.

TheOREM A. If $P(z)$ is a polynomial of degree $n$ with $\operatorname{Max}_{|z|-1}|P(z)|=1$ and $P(z)$ has no zeros in the disk $|z|<1$, then

$$
\operatorname{Max}_{|z|=R>1}|P(z)|<\frac{R^{n}+1}{2} \text {. }
$$

The result is best possible and equality in (3) holds for $P(z)=\left(z^{n}+1\right) / 2$.

We shall generalize Theorem A by proving the following theorems.

TheOREM 1. If $P(z)$ is a polynomial of degree $n$ with $\operatorname{Max}_{|z|-1}|P(z)|=1$ and $P(z)$ has no zeros in the disk $|z|<k$ where $K>1$, then

$$
\operatorname{Max}_{|z|=R>1}|P(z)|<\frac{\left(R^{n}+1\right)(R+k)^{n}}{(R+k)^{n}+(1+R k)^{n}} .
$$

Theorem 1 is a generalization of Theorem $\mathbf{A}$ in a compact form but unfortunately with the exception of $n=1$, (4) does not appear to be sharp for $k>1$.

However, a precise estimate is given by the following theorem.

Received by the editors December 17, 1979 and, in revised form, March 27, 1980.

AMS (MOS) subject classifications (1970). Primary 30A06, 30A64; Secondary 26A82.

Key words and phrases. Growth of maximum modulus, inequalities for polynomials. 
TheOREM 2. If $P(z)$ is a polynomial of degree $n$ with $\operatorname{Max}_{|z|-1}|P(z)|=1$ and $P(z)$ has no zeros in the disk $|z|<k$ where $k \geqslant 1$, then for $R>1$ we have

$$
\operatorname{Max}_{|z|=R}|P(z)| \leqslant \frac{R^{n}+k^{n}}{1+k^{n}} \text { for } R \geqslant k^{2}
$$

and

$$
\operatorname{Max}_{|z|=R}|P(z)| \leqslant \frac{(R+k)^{n}}{(1+k)^{n}} \quad \text { for } 1<R<k^{2} .
$$

The result is best possible with equality in (5) for $P(z)=\left(z^{n}+k^{n}\right) /\left(1+k^{n}\right)$ and in (6) for $P(z)=(z+k)^{n} /(1+k)^{n}$.

For the proofs of these theorems we need the following Lemma.

LEMMA. If $P(z)$ is a polynomial of degree $n$ with $\operatorname{Max}_{|z|-1}|P(z)|=1$, then

where $Q(z)=z^{n} \frac{\left|P\left(R e^{i \theta}\right)\right|+\left|Q\left(\operatorname{Re}^{i \theta}\right)\right|}{P(1 / \bar{z})}$ and $R>1$.

Proof of the Lemma. Since $|P(z)|<1$ for $|z|=1$, therefore, if $\alpha$ is a complex number such that $|\alpha|>1$, it follows from Rouche's theorem that the polynomial $F(z)=P(z)-\alpha$ does not vanish inside the unit circle. Thus the polynomial

$$
G(z)=z^{n} \overline{F(1 / \bar{z})}=z^{n} \overline{P(1 / \bar{z})}-\bar{\alpha} z^{n}=Q(z)-\bar{\alpha} z^{n}
$$

has all its zeros in $|z|<1$ and $|F(z)|=|G(z)|$ for $|z|=1$. Hence $|G(z) / F(z)|$ is analytic on and inside the unit circle, and on the boundary $|G(z) / F(z)|=1$. By the Maximum Modulus Principle it follows that $|G(z)|<|F(z)|$ for $|z|<1$. Replacing $z$ by $1 / \bar{z}$ we get $\left|z^{n} \overline{G(1 / \bar{z})}\right|<\left|z^{n} \overline{F(1 / \bar{z})}\right|$ for $|z|>1$. Since $z^{n} \overline{G(1 / \bar{z})} \equiv F(z)$, we conclude that $|F(z)| \leqslant|G(z)|$ for $|z| \geqslant 1$. Therefore, in particular

$$
\left|F\left(R e^{i \theta}\right)\right| \leqslant\left|G\left(R e^{i \theta}\right)\right| \text { where } R \geqslant 1 \text { and } 0<\theta<2 \pi \text {. }
$$

This gives

$$
\left|P\left(\operatorname{Re}^{i \theta}\right)-\alpha\right|<\left|Q\left(R e^{i \theta}\right)-\bar{\alpha} R^{n} e^{i n \theta}\right|, \quad 0<\theta<2 \pi .
$$

Now choosing an argument of $\alpha$ such that $\left|Q\left(R e^{i \theta}\right)-\bar{\alpha} R^{n} e^{i n \theta}\right|=|\alpha| R^{n}-$ $\left|Q\left(R e^{i \theta}\right)\right|$, we obtain

$$
\left|P\left(R e^{i \theta}\right)\right|-|\alpha|<|\alpha| R^{n}-\left|Q\left(R e^{i \theta}\right)\right|, \quad 0<\theta<2 \pi .
$$

Equivalently

$$
\left|P\left(\operatorname{Re}^{i \theta}\right)\right|+\left|Q\left(\operatorname{Re}^{i \theta}\right)\right|<|\alpha|\left(R^{n}+1\right), \quad 0<\theta<2 \pi
$$

Letting now $|\alpha| \rightarrow 1$, we obtain (7) and this proves the Lemma.

Proof of Theorem 1. Since the polynomial $P(z)$ has all its zeros in $|z|>k>1$, we write

$$
P(z)=\prod_{j=1}^{n}\left(z-r_{j} e^{i \theta_{j}}\right) \quad \text { where } r_{j}>k, j=1,2, \ldots, n .
$$


Then $Q(z)=z^{n} \overline{P(1 / \bar{z})}=\Pi_{j=1}^{n}\left(1-z r_{j} e^{-i \theta}\right)$ so that clearly we have for $0<\theta<2 \pi$ and for $R>1$

$$
\begin{aligned}
\left|P\left(\operatorname{Re}^{i \theta}\right) / Q\left(\operatorname{Re}^{i \theta}\right)\right| & =\prod_{j=1}^{n}\left|\left(\operatorname{Re}^{i \theta}-r_{j} e^{i \theta}\right) /\left(1-\operatorname{Rr}_{j} e^{i\left(\theta-\theta_{j}\right)}\right)\right| \\
& =\prod_{j=1}^{n}\left|\left(\operatorname{Re} e^{i\left(\theta-\theta_{j}\right)}-r_{j}\right) /\left(1-r_{j} \operatorname{Re}^{i\left(\theta-\theta_{j}\right)}\right)\right| \\
& <\prod_{j=1}^{n}\left(R+r_{j}\right) /\left(1+R r_{j}\right) \\
& <\prod_{j=1}^{n}(R+k) /(1+R k)=(R+k)^{n} /(1+R k)^{n} .
\end{aligned}
$$

This implies

$$
\left((1+R k)^{n} /(R+k)^{n}\right)\left|P\left(R e^{i \theta}\right)\right|<\left|Q\left(R e^{i \theta}\right)\right|, \quad 0<\theta<2 \pi .
$$

Combining this with the conclusion of the Lemma we obtain

$$
\left(1+(1+R k)^{n} /(R+k)^{n}\right)\left|P\left(R e^{i \theta}\right)\right|<R^{n}+1, \quad 0<\theta<2 \pi .
$$

Consequently

$$
\operatorname{Max}_{|z|=R>1}|P(z)|<\frac{\left(R^{n}+1\right)(R+k)^{n}}{(R+k)^{n}+(1+R k)^{n}},
$$

which is the desired result.

Proof of Theorem 2. Since all the zeros of $P(z)$ lie in $|z|>k>1$, we write as before

$$
\begin{aligned}
& P(z)=\prod_{j=1}^{n}\left(z-r_{j} e^{i \theta_{j}}\right) \quad \text { where } r_{j}>k, j=1,2, \ldots, n . \\
& \text { Then } Q(z)=z^{n} \overline{P(1 / \bar{z})}=\prod_{j=1}^{n}\left(1-z r_{j} e^{-i \theta_{\theta}}\right) . \text { Now } \\
&\left|P\left(k^{2} e^{i \theta}\right) / Q\left(e^{i \theta}\right)\right|=\prod_{j=1}^{n}\left|\left(k^{2} e^{i \theta}-r_{j} e^{i \theta}\right) /\left(1-r_{j} e^{i\left(\theta-\theta_{j}\right)}\right)\right| \\
&=\prod_{j=1}^{n}\left|\left(k^{2} e^{i\left(\theta-\theta_{j}\right)}-r_{j}\right) /\left(1-r_{j} e^{i\left(\theta-\theta_{j}\right)}\right)\right| \\
& \prod_{j=1}^{n} k=k^{n} \text { for } 0<\theta<2 \pi .
\end{aligned}
$$

Therefore, we have

$$
\left|P\left(k^{2} z\right)\right|<k^{n}|Q(z)| \text { for }|z|=1 .
$$

Since all the zeros of $Q(z)$ lie in $|z|<1 / k<1$, it follows from the Maximum Modulus Principle that

$$
\left|P\left(k^{2} z\right)\right|<k^{n}|Q(z)| \text { for }|z|>1 .
$$

Hence in particular we have

$$
\left|P\left(k^{2} R e^{i \theta}\right)\right|<k^{n}\left|Q\left(R e^{i \theta}\right)\right| \text { for } R>1 \text { and } 0<\theta<2 \pi .
$$


This inequality with the help of the Lemma gives

$$
\left|P\left(k^{2} R e^{i \theta}\right)\right|+k^{n}\left|P\left(R e^{i \theta}\right)\right|<k^{n}\left(R^{n}+1\right), \quad 0<\theta<2 \pi .
$$

Now for every given $\theta, 0<\theta<2 \pi$, and for $R>1$, we have either

$$
\left|P\left(k^{2} \operatorname{Re}^{i \theta}\right)\right|-\left|P\left(R e^{i \theta}\right)\right|<\left(k^{n}-1\right) R^{n}
$$

or

$$
\left|P\left(k^{2} R e^{i \theta}\right)\right|-\left|P\left(R e^{i \theta}\right)\right|>\left(k^{n}-1\right) R^{n} .
$$

Inequality (10) yields with the help of inequality (9)

$$
\left(1+k^{n}\right)\left|P\left(k^{2} R e^{i \theta}\right)\right|<\left(k^{2 n} R^{n}+k^{n}\right)
$$

and inequality (11) yields with the help of inequality (9)

$$
\begin{gathered}
\left(1+k^{n}\right)\left|P\left(R e^{i \theta}\right)\right|<\left|P\left(k^{2} \operatorname{Re}^{i \theta}\right)\right|+k^{n}\left|P\left(R e^{i \theta}\right)\right|-\left(k^{n}-1\right) R^{n} \\
<k^{n}\left(R^{n}+1\right)-\left(k^{n}-1\right) R^{n}=R^{n}+k^{n} .
\end{gathered}
$$

Thus we have either

$$
\underset{|z|=R}{\operatorname{aax}}|P(z)|<\left(R^{n}+k^{n}\right) /\left(1+k^{n}\right) \quad \text { for } R>k^{2}
$$

or

$$
\operatorname{Max}_{|z|=R}|P(z)|<\left(R^{n}+k^{n}\right) /\left(1+k^{n}\right) \text { for } R>1 .
$$

From (12) and (13), it follows that, we have in any case

$$
\operatorname{Max}_{|z|=R}|P(z)|<\frac{R^{n}+k^{n}}{1+k^{n}} \quad \text { for } R>k^{2}
$$

and this proves the inequality (5).

To prove the inequality (6), we observe that for $1<R<k^{2}$ and $0<\theta<2 \pi$

$$
\begin{aligned}
\left|P\left(R e^{i \theta}\right) / P\left(e^{i \theta}\right)\right| & =\prod_{j=1}^{n}\left|\left(R e^{i \theta}-r_{j} e^{i \theta_{j}}\right) /\left(e^{i \theta}-r_{j} e^{i \theta}\right)\right| \\
& =\prod_{j=1}^{n}\left|\left(R e^{i\left(\theta-\theta_{j}\right)}-r_{j}\right) /\left(e^{i\left(\theta-\theta_{j}\right)}-r_{j}\right)\right| \\
& <\prod_{j=1}^{n}\left(R+r_{j}\right) /\left(1+r_{j}\right) \\
& <\prod_{j=1}^{n}(R+k) /(1+k)=(R+k)^{n} /(1+k)^{n} .
\end{aligned}
$$

Consequently

$$
\operatorname{Max}_{|z|=R}|P(z)|<\frac{(R+k)^{n}}{(1+k)^{n}} \quad \text { for } 1<R<k^{2}
$$

This is the inequality (6) and the theorem is completely established. 
ACKNOWLEDgement. The authors are highly thankful to the referee for his help and encouragement.

\section{REFERENCES}

1. N. C. Ankeny and T. J. Rivlin, On a theorem of S. Bernstein, Pacific J. Math. 5 (1955), 849-852.

2. P. D. Lax, Proof of a conjecture of P. Erdös on the derivative of a polynomial, Bull. Amer. Math. Soc. 50 (1944), 509-513.

3. G. Polya and G. Szegö, Aufgaben und Lehrsätze aus der Analysis, Springer-Verlag, Berlin, 1925.

4. M. Riesz, Über einen Satz des Herra Serge Bernstein, Acta Math. 40 (1916), 337-349.

Post Graduate Department of Mathematics, University of Kashmir, Hazratbal, Srinagar 190006, INDIA 